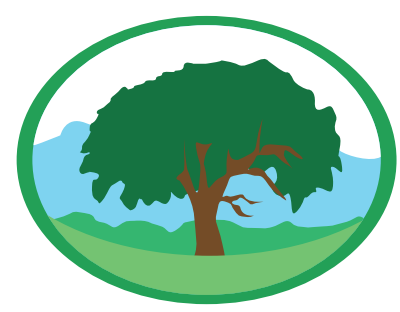

\title{
Degradação fotocatalítica de ciprofloxacina usando óxido de zinco em espuma vítrea
}

\author{
CARVALHO, C.0. ${ }^{1}$, ARSAND, D. R. ${ }^{2}$, MACHADO, F. M. ${ }^{3}$, RANGEL, E. M. ${ }^{4}$, MELO, C. C. N.$^{5}$ \\ 'Universidade Federal de Pelotas - Centro de Desenvolvimento Tecnológico/CDTec - Pelotas/RS, Mestranda em Ciência e \\ Engenharia de Materiais - carolineoli.cardgmail.com \\ ${ }^{2}$ Instituto Federal Sul-rio-grandense - Programa de Pós-graduação em Engenharia e Ciências Ambientais (PPGECA) - \\ danielarsandapelotas.ifsul.com.br \\ ${ }^{3}$ Universidade Federal de Pelotas - Centro de Desenvolvimento Tecnológico/CDTec - Pelotas/RS, Docente do Programa de Pós \\ Graduação em Ciência e Engenharia de Materiais/PPGCEM - fernando.machado.machado80agmail.com \\ «Universidade Federal de Pelotas - Centro de Desenvolvimento Tecnológico/CDTec - Pelotas/RS, Doutoranda em Ciência e \\ Engenharia de Materiais - eduardamrangeldgmail.com \\ ${ }_{5}^{5}$ Universidade Federal de Pelotas - Centro de Desenvolvimento Tecnológico/CDTec - Pelotas/RS, Mestrando em Ciência e \\ Engenharia de Materiais - caio12cnmahotmail.com
}

Palavras-chave: Fotocatálise heterogênea, fotodegradação, catalisador, antibióticos.

\begin{abstract}
Resumo
Neste trabalho é apresentada a fotodegradação do antibiótico ciprofloxacina pela aplicação de espuma vítrea fotocatalisadora, produzida a partir de óxido de zinco (espécie fotoativa), vidro residual de lâmpadas fluorescentes (matriz vítrea) e carbonato de cálcio lagente espumante). 0 vidro de resíduo precursor teve a sua composição química e estrutura cristalina elucidadas através das técnicas de fluorescência de raios- $X$ e difração de raios- $X$, respectivamente. A espuma vítrea fotocatalisadora foi formulada com $5 \%$ em massa de agentes espumantes e $10 \%$ em massa de $\mathrm{ZnO}$, conformada por prensagem uniaxial e queimada à 800 ${ }^{\circ} \mathrm{C}$, com taxa de aquecimento de $2,5{ }^{\circ} \mathrm{C} \mathrm{min}{ }^{-1}$ e 30 min de patamar. Os testes de fotodegradação foram realizados em fotorreator equipado com lâmpada de vapor de mercúrio de baixa pressão (6 W) imersa na solução; e 0,5 $L$ de solução de trabalho $0,1 \mathrm{~g} \mathrm{~L}^{-1}$ ciprofloxacina. Os resultados mostraram que a degradação do antibiótico aplicando espuma vítrea fotocatalisadora/UV foi aproximadamente 2,5 vezes mais eficiente do que o uso de somente radiação ultravioleta. Além disso, o material produzido mostra uma alternativa viável para a destinação de resíduos de lâmpadas fluorescentes.
\end{abstract}

\section{PHOTOCATALYTIC DEGRADATION OF CIPROFLOXACIN USING ZINC OXIDE IN FOAM GLASS}

Keywords: Heterogeneous photocatalysis, photodegradation, catalyst, antibiotic.

\begin{abstract}
This work brings forward the photodegradation of the antibiotic ciprofloxacin by the application of photocatalytic foam glass, produced from zinc oxide (photoactive species), residual fluorescent lamp glass (vitreous matrix) and calcium carbonate (foaming agent). The precursor residue glass had its chemical composition and crystalline structure elucidated by X-ray fluorescence and X-ray diffraction techniques, respectively. The photocatalytic foam glass was formulated with $5 \%$ by weight of foaming agent and $10 \%$ by mass of $\mathrm{ZnO}$, formed by uniaxial pressing and burned at $800{ }^{\circ} \mathrm{C}$, with a heating rate of $2.5^{\circ} \mathrm{C} \mathrm{min}-1$ and $30 \mathrm{~min}$ of the plateau. The photodegradation tests were performed on a photoreactor equipped with a low-pressure mercury vapor lamp (6 W) immersed in the solution; and $0.5 \mathrm{~L}$ of working solution $0.1 \mathrm{~g} \mathrm{~L}^{-1}$ of ciprofloxacin. The results show that the degradation of the antibiotic using foam glass/UV was approximately 2.5 times more efficient
\end{abstract}


than the use of ultraviolet radiation only. In addition, the material produced shows a viable alternative for the disposal of residues from fluorescent lamps.

\section{INTRODUÇÃO}

Antibióticos são administrados em larga escala para o tratamento de doenças na medicina humana e animal (SOLLIEC et al., 2016). Nessa classe de composto farmacêutico se destaca a ciprofloxacina (CIP) (SUN et al., 2016). Como consequência desse elevado consumo, é recorrente a presença da CIP em meios hídricos (PRUTTHIWANASAN et al., 2016).

Técnicas e processos que mitiguem a presença dessa substância no ambiente são de grande interesse, uma vez que sua presença em corpos hídricos, mesmo em baixas concentrações, causam a seleção de bactérias resistentes a antibióticos, ocasionando desequilíbrios nos níveis tróficos (CHATZITAKIS et al., 2008; LAPWORTH et al., 2012). Com isso, diversos autores apresentam alternativas para a remoção ou degradação de CIP em meio aquoso, dentre elas a adsorção (WANG et al., 2015; KYZAS et al., 2016), a eletrocoagulação (ARSAND et al., 2013; BRILLAS et al., 2015) e principalmente, a fotocatálise (YAN et al., 2013; HUO et al., 2016). A fotocatálise heterogênea é uma técnica oxidativa avançada que apresenta como vantagem a degradação de poluentes/contaminantes, sem que ocorra a transferência do contaminante de uma fase para outra (MOURÃO et al., 2009; MERG et al., 2010). Mas essa técnica apresenta como limitante a necessidade de uma etapa de filtração ao final do processo (SHEN et al., 2012). Para que isso seja evitado, suportes para fotocatalisadores são usados como alternativa (COLEMAN et al., 2004; RAY et al., 2016). Entretanto, estudos com o uso de espumas vítreas como suporte para fotocatalisadores para remoção de fármacos não é encontrado.

Neste contexto, o objetivo deste trabalho é estudar a degradação de CIP em meio aquoso através da fotocatálise utilizando espuma vítrea fotocatalisadora (EVF) tendo $\mathrm{ZnO}$ como espécie fotoativa.

\section{MATERIAIS E MÉTODOS}

\section{Antibiótico e Materiais Precursores}

O princípio ativo CIP (CAS: 86393-32-0,
$\left.\mathrm{C}_{17} \mathrm{H}_{18} \mathrm{FN}_{3} \mathrm{O}_{3}, \mathrm{MM}: 331,34 \mathrm{~g} \mathrm{~mol}^{-1}\right)$, um antibiótico do grupo das fluorquinolonas (Figura 1), foi obtido comercialmente.

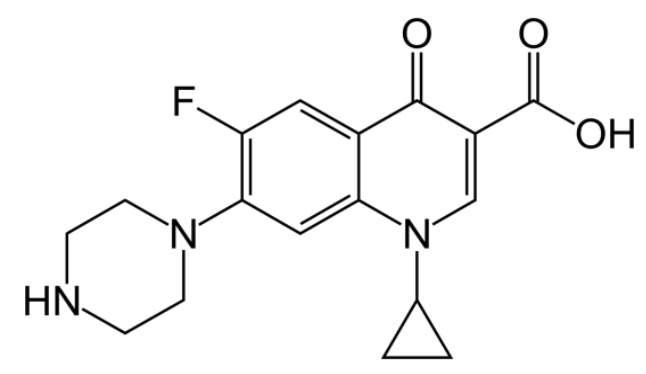

Figura 1. Estrutura molecular da Ciprofloxacina.

Como materiais precursores da EVF foram utilizados: vidro de resíduo de lâmpada fluorescente (previamente descontaminado) como matriz vítrea; carbonato de cálcio (DINÂMICA, 99\% de pureza) como agente espumante; e óxido de zinco (VETEC, 99\% de pureza) como espécie fotoativa. O $\mathrm{ZnO}$ foi escolhido por ser um semicondutor barato e com ótimas propriedades optoeletrônicas, tendo band gap de aproximadamente 3,36 eV (SÁNCHEZ et al., 2013).

O vidro de resíduo precursor teve a sua composição química e estrutura cristalina elucidadas através das técnicas de fluorescência de raios-X (Shimatzu, modelo XRF1800) e difração de raios-X (Bruker, modelo D2 PHASER) equipado com ânodo de cobre, operado a $30 \mathrm{kV}$ e $10 \mathrm{~mA}$, operando com os seguintes parâmetros de ensaio: intervalo angular de varredura $10^{\circ}<2 \theta<80^{\circ}$ com passos de $0,05^{\circ}$, por um período de 1 s para cada passo.

\section{Produção da espuma vítrea fotocatalisadora}

As espumas vítreas utilizadas foram produzidas segundo metodologia proposta por Pokorny et al. (2008) com adição de $\mathrm{ZnO}$ como substância fotoativa, dando origem a EVF. Essas espumas foram confeccionadas com pó de vidro com granulometria \#100, 5\% (m/m) de $\mathrm{CaCO}_{3}$ e $10 \%(\mathrm{~m} / \mathrm{m})$ de $\mathrm{ZnO}$. A mistura foi homogeneizada e, então, adicionado 8\% em massa de uma solução de água destilada e álcool polivinílico (DINÂMICA, 99\% de pureza). Por seguinte, o material foi conformado em moldes cilíndricos com $5 \mathrm{~cm}$ de diâmetro e $10 \mathrm{~cm}$ de altura. Os corpos de prova foram conformados em prensa 
hidráulica (EMIC, modelo PC200C) a $40 \mathrm{MPa}$. O material resultante foi seco em ambiente livre por $24 \mathrm{~h}$ e posteriormente em estufa a $100 \pm 5{ }^{\circ} \mathrm{C}$ por $24 \mathrm{~h}$. Por fim, os corpos de prova foram queimados em forno elétrico tipo mufla à $800^{\circ} \mathrm{C}$, com taxa de aquecimento de $2,5^{\circ} \mathrm{C} \min ^{-1}$ e 30 min de patamar.

\section{Testes de Fotodegradação}

Os testes de fotodegradação foram realizados usando fotorreator homemade de $600 \mathrm{~mL}$ (Figura 2(a)). Como fonte de radiação UV-C foi usada uma lâmpada de vapor de mercúrio de baixa pressão, com 6 W de potência, imersa na soluçáo com auxílio de tubo de quartzo. Por questóes de segurança o fotorreator foi blindado externamente para evitar fugas da radiação UV. A eficiência da fotodegradação de CIP com e sem o uso de EVF foi avaliada através de duas condiçóes experimentais: com o uso de luz UV-C, somente; e com o uso de radiação UV combinada com a EVF. Para ambos os testes as demais condiçóes e tempos de irradiação foram mantidos.

No experimento com a aplicação do material produzido, duas EVF na forma de pastilha (Figura 2, indicação: 2), com aproximadamente $20 \mathrm{~cm}^{2}$ de área fotoativa cada foram usadas; a distância entre as EVFs foi de $8 \mathrm{~cm}$.
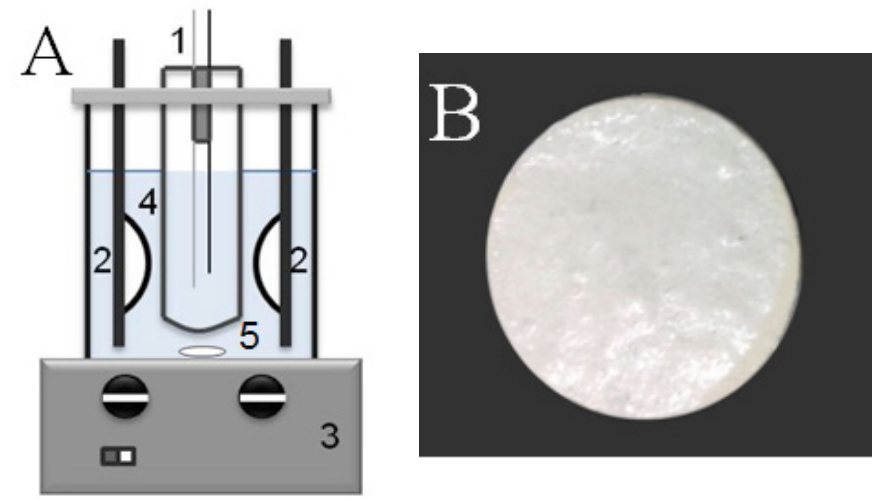

Figura 2. (A) Reator fotocatalítico homemade, onde 1: Lâmpada de radiação UV, 2: EVFs, 3: Agitador magético, 4: Solução com a CIP, 5: Barra magnética e (B) imagem da EVF.

\section{Fotodegradação de Ciprofloxacina}

Para avaliar a degradaçáo de CIP, em ambos os testes foram utilizadas soluções com concentração de $0,1 \mathrm{~g} \mathrm{~L}^{-1}$ de CIP e amostras foram coletadas $(2 \mathrm{~mL}) \mathrm{em}$ triplicata nos tempos: $0,5,10,15,30,60$ e $120 \mathrm{~min}$. As amostras foram armazenadas a $4 \pm 1{ }^{\circ} \mathrm{C}$ na ausência de luz até determinação da concentração de CIP por espectrofotometria usando metodologia proposta por Fratini (1996). As análises foram feitas utilizando o espectrofotômetro UV-VIS (Biospectro, modelo 220b). As determinações foram realizadas usando comprimento de onda de trabalho de $334 \mathrm{~nm}$. A eficiência da fotodegradação de CIP foi determinada a partir da porcentagem de degradação do fármaco, calculada através da Equação 1.

$$
\text { Degradação(\%)=100x } \frac{\left(C_{o}-C_{f}\right)}{C_{o}}
$$

onde, $\mathrm{C}_{\mathrm{o}}$ representa a concentração inicial $\left(\mathrm{mg} \mathrm{L}^{-1}\right)$ de CIP em solução e $\mathrm{C}_{\mathrm{f}}$ a concentração final $\left(\mathrm{mg} \mathrm{L}^{-1}\right)$.

\section{RESULTADOS E DISCUSSÃO}

\section{Caracterização do Vidro de Resíduo Precursor}

A Tabela 1 apresenta a composição química do resíduo de lâmpada fluorescente utilizada como material precursor na fabricação da EVF. O material analisado possui elementos típicos de um vidro sodocálcico, com dióxido de silício como componente principal, seguido por óxido de cálcio e menores concentraçôes de outros elementos. Pode-se perceber, também, a descontaminação completa do material de mercúrio. A ausência do metal é necessária para evitar possíveis contaminações no momento da fabricação da espuma vítrea, evitando assim danos à saúde (HA et al., 2017).

Tabela 1. Composição química do vidro de lâmpada fluorescente.

\begin{tabular}{|l|l|}
\hline Analito & $\begin{array}{l}\text { Composicão } \\
\text { química }[\%]\end{array}$ \\
\hline $\mathrm{SiO}_{2}$ & 76,44 \\
\hline $\mathrm{CaO}$ & 11,63 \\
\hline $\mathrm{Na}_{2} \mathrm{O}$ & 2,45 \\
\hline $\mathrm{Al}_{2} \mathrm{O}_{3}$ & 1,94 \\
\hline $\mathrm{K}_{2} \mathrm{O}$ & 1,86 \\
\hline $\mathrm{PbO}$ & 1,05 \\
\hline $\mathrm{BaO}$ & 1,02 \\
\hline $\mathrm{MgO}$ & 0,91 \\
\hline $\mathrm{P}_{2} \mathrm{O}_{5}$ & 0,86 \\
\hline $\mathrm{Fe}_{2} \mathrm{O}_{3}$ & 0,83 \\
\hline $\mathrm{SO}_{3}$ & 0,39 \\
\hline
\end{tabular}




\begin{tabular}{|l|l|}
\hline $\mathrm{Y} 2 \mathrm{O} 3$ & 0,26 \\
\hline $\mathrm{SrO}$ & 0,25 \\
\hline $\mathrm{ZrO}_{2}$ & 0,09 \\
\hline
\end{tabular}

$\mathrm{O}$ difratograma de raios- $\mathrm{X}$ da amostra de vidro de lâmpada fluorescente é apresentado na Figura 3. Podese visualizar uma banda larga entre $20^{\circ}<2 \theta<40^{\circ}$, típica de material amorfo (MUGONI et al., 2015).

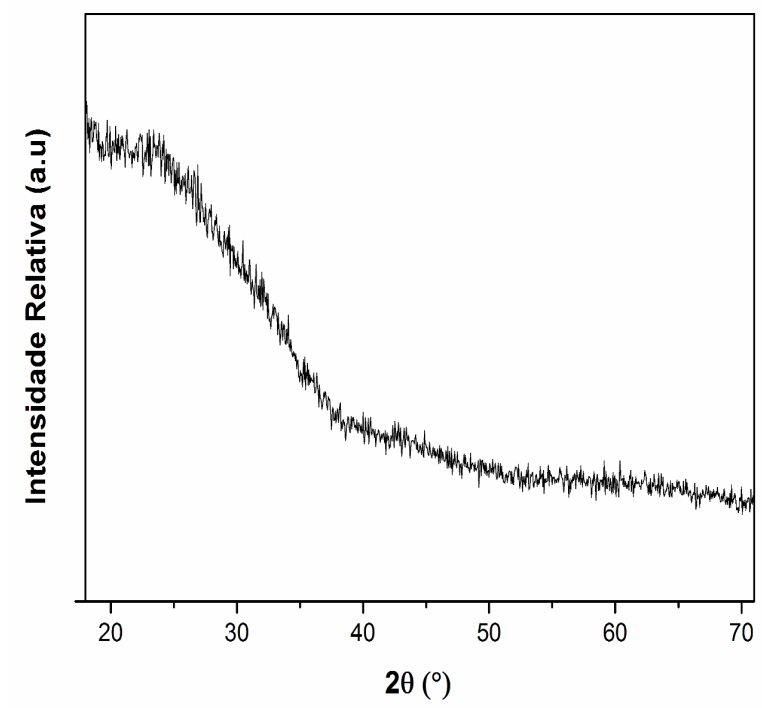

Figura 3. Difratograma de raios-X do vidro de resíduo de lâmpada fluorescente.

\section{Degradação de Ciprofloxacina}

As degradaçốes resultantes dos experimentos de fotólise e dos experimentos usando EVF estão demonstradas na Figura 4.

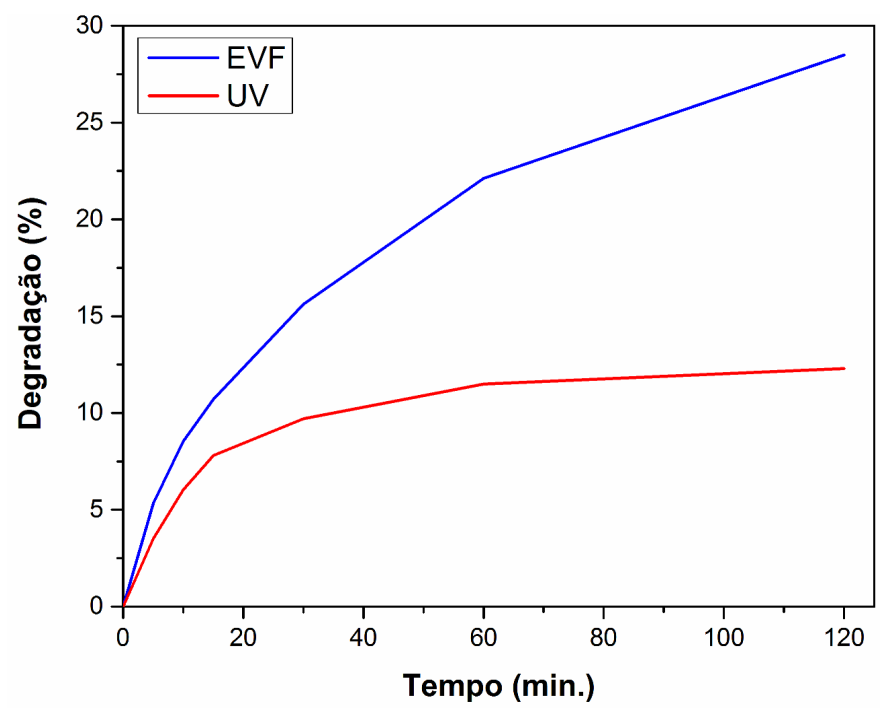

Figura 4. Porcentagem de remoção de ciprofloxacina utilizando fotocatálise combinada com EVF e apenas fotólise.

Os resultados mostram que a fotodegradação da
CIP usando EVF foi maior quando comparada a fotodegradação somente irradiada com luz UV. Os experimentos aplicando EVF apresentaram 28\% de degradação em 120 minutos de experimento, enquanto os experimentos de fotólise alcançaram $12 \%$ de remoção de CIP no mesmo período de tempo de exposição à radiação UV, representando uma degradação aproximadamente 2,5 vezes maior. Desta forma, pode-se perceber que o uso da EVF contendo o $\mathrm{ZnO}$ como espécie fotoativa alcança melhores resultados de degradaçáo quando comparado com a aplicação de somente energia radiante na degradação de CIP, comprovando assim a participação do catalisador no processo de degradação do fármaco, mostrando seu uso potencial na degradaçáo de CIP em meios aquosos.

\section{CONCLUSÕES}

Com base nos resultados pode-se concluir que a CIP é degradada em meio aquoso e que o uso de EVF/UV-C mostrou maior efetividade na degradação do antibiótico em solução aquosa. Os resultados mostraram que a fotodegradação do composto é aproximadamente 2,5 vezes mais eficiente quando aplicado espumas vítrea fotocatalisadora tendo o $\mathrm{ZnO}$ como espécie fotoativa, comprovando a participação desse semicondutor na reação. Ainda, a EVF demonstrou ser inovadora e sustentável, podendo substituir substâncias tradicionalmente utilizadas em fotocatálises heterogêneas na forma de suspensão, eliminando a etapa de separação de fases ao final da reação e tornando a fotodegradação catalisada de CIP viável, menos morosa e de baixo custo.

\section{REFERÊNCIAS}

ARSAND, D. R.; KÜMMERER, K.; MARTINS, A. F. Removal of dexamethasone from aqueous solution and hospital wastewater by electrocoagulation. The Science of the total environment, v. 443, p. 351-357, 15 jan. 2013.

BRILLAS, E.; ENRIC, B.; IGNASI, S. Electrochemical removal of pharmaceuticals from water streams: Reactivity elucidation by mass spectrometry. Trends in analytical chemistry: TRAC, v. 70, p. 112-121, 2015.

CHATZITAKIS, A. et al. Photocatalytic degradation and drug activity reduction of Chloramphenicol. Water research, v. 42, n. 1-2, p. 386-394, jan. 2008.

COLEMAN, H. M. et al. Rapid loss of estrogenicity of steroid estrogens by UVA photolysis and photocatalysis over an 
immobilised titanium dioxide catalyst. Water research, v. 38, n. 14-15, p. 3233-3240, 2004.

FRATINI, L. Ciprofloxacin determination by visible light spectrophotometry using iron(III)nitrate. International journal of pharmaceutics, v. 127, n. 2, p. 279-282, 1996.

HA, E. et al. Current progress on understanding the impact of mercury on human health. Environmental research, v. 152, p. 419-433, jan. 2017.

HUO, P. et al. Fabrication of ZnWO4-CdS heterostructure photocatalysts for visible light induced degradation of ciprofloxacin antibiotics. Journal of Industrial and Engineering Chemistry, v. 37, p. 340-346, 2016.

KYZAS, George Z.; DELIYANNI, Eleni A.; MATIS, Kostas A. Activated carbons produced by pyrolysis of waste potato peels: Cobalt ions removal by adsorption. Colloids and Surfaces A: Physicochemical and Engineering Aspects, v. 490, p. 74-83, 2016.

LAPWORTH, D. J. et al. Emerging organic contaminants in groundwater: A review of sources, fate and occurrence. Environmental pollution, v. 163, p. 287-303, 2012.

MERG, J. C. et al. Incorporação de dióxido de titânio em zeólitas para emprego em fotocatálise heterogênea. Química Nova, v. 33, n. 7, p. $1525-1528,2010$.

MOURÃO, H. A. J. L. et al. Nanoestruturas em fotocatálise: uma revisão sobre estratégias de síntese de fotocatalisadores em escala nanométrica. Química Nova, v. 32, n. 8, p. 2181-2190, 2009.

MUGONI, C. et al. Design of glass foams with low environmental impact. Ceramics International v. 41 p. 3400-3408, 2015

POKORNY, A.; VICENZI, J.; BERGMANN, C. P. Influência da adiçáo de alumina na microestrutura da espuma vítrea. Cerâmica, v. 54, n. 329, p. 97-102, 2008.
PRUTTHIWANASAN, B et al. Fluorescent labelling of ciprofloxacin and norfloxacin and its application for residues analysis in surface water. Talanta, v. 159, p. 74-79, 1 out. 2016. RAY, S.; SRIMANTA, R.; LALMAN, J. A. Fabrication and characterization of an immobilized titanium dioxide (TiO2) nanofiber photocatalyst. Materials Today: Proceedings, v. 3, n. 6, p. 1582-1591, 2016.

SÁNCHEZ, F. A. L. et al. Photocatalytic activity of nanoneedles, nanospheres, and polyhedral shaped $\mathrm{ZnO}$ powders in organic dye degradation processes. Journal of Alloys and Compounds, v. 572, p. 68-73, 2013.

SHEN, C. et al. Facile synthesis and photocatalytic properties of $\mathrm{TiO} 2$ nanoparticles supported on porous glass beads. Chemical engineering journal , v. 209, p. 478-485, 2012.

SOLLIEC, M. et al. Fractionation and analysis of veterinary antibiotics and their related degradation products in agricultural soils and drainage waters following swine manure amendment. The Science of the total environment, v. 543, p. 524-535, 2016.

SUN, Y. et al. Characterization and ciprofloxacin adsorption properties of activated carbons prepared from biomass wastes by H3PO4 activation. Bioresource technology, v. 217, p. 239244, out. 2016.

WANG, Y. X.; NGO, H. H.; GUO, W. S. Preparation of a specific bamboo based activated carbon and its application for ciprofloxacin removal. The Science of the total environment, v. 533, p. 32-39, 2015.

YAN, Y. et al. Microwave-assisted in situ synthesis of reduced graphene oxide-BiVO4 composite photocatalysts and their enhanced photocatalytic performance for the degradation of ciprofloxacin. Journal of hazardous materials, v. 250-251, p. 106-114, 15 abr. 2013. 